\title{
Vinyl-fluorene Molecular Wires for Voltage Imaging with Enhanced Sensitivity and Reduced Phototoxicity
}

\author{
Steven C. Boggess ${ }^{\ddagger}$, Shivaani S. Gandhi ${ }^{\ddagger}$, and Evan W. Miller ${ }^{\ddagger \S}{ }^{\star *}$ \\ Departments of ${ }^{\ddagger}$ Chemistry and ${ }^{\S}$ Molecular \& Cell Biology and ${ }^{\dagger}$ Helen Wills Neuroscience Institute. University of \\ California, Berkeley, California 94720, United States.
}

\begin{abstract}
Fluorescent voltage indicators are an attractive alternative for studying the electrical activity of excitable cells; however, the development of indicators that are both highly sensitive and low in toxicity over long-term experiments remains a challenge. Previously, we reported a fluorene-based voltage-sensitive fluorophore that exhibits much lower phototoxicity than previous voltage indicators in cardiomyocyte monolayers, but suffers from low sensitivity to membrane potential changes. Here, we report that the addition of a single vinyl spacer in the fluorene molecular wire scaffold improves the voltage sensitivity 1.5 - to 3.5-fold over fluorene-based voltage probes. Furthermore, we demonstrate the improved ability

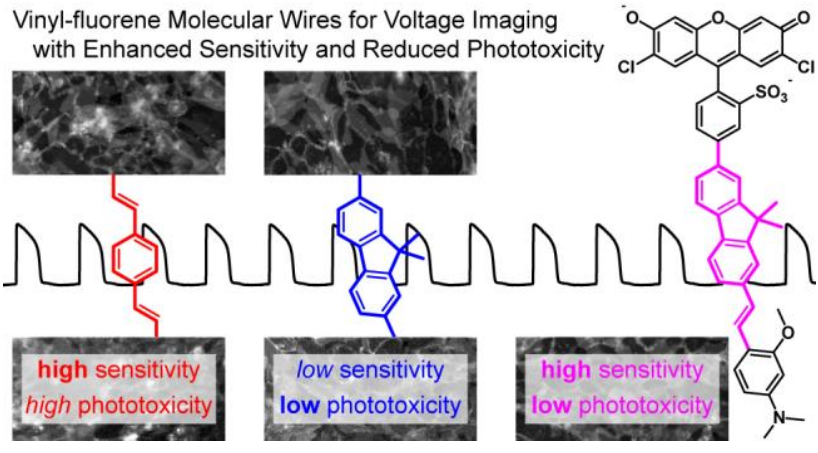
of the new vinyl-fluorene VoltageFluors (v-fVFs) to monitor action potential kinetics in both mammalian neurons and human induced pluripotent stem cell-derived cardiomyocytes (hiPSC-CMs). Addition of the vinyl spacer between the aniline donor and fluorene monomer results in indicators that are significantly less phototoxic in cardiomyocyte monolayers. These results demonstrate how structural modification to the voltage sensing domain have a large effect on improving the overall properties of molecular wire-based voltage indicators.
\end{abstract}

Cells expend a large proportion of their energy budget to maintain tight control over the electrical potential across the plasma membrane. In excitable cells, membrane potential $\left(\mathrm{V}_{\mathrm{mem}}\right)$ can change quickly, and these action potentials govern the unique physiology of neurons and cardiomyocytes. Patch-clamp electrophysiology is the goldstandard for measuring electrical impulses in live cells, but is highly invasive and low throughput. ${ }^{1}$ To complement traditional electrophysiology, we have been developing voltage-sensitive fluorophores, or VoltageFluors, that can optically measure rapid changes of $\mathrm{V}_{\text {mem }}$ in live cells. ${ }^{2}$ Using fluorescence to monitor $V_{\text {mem }}$ dynamics is attractive because it circumvents many of the issues associated with electrode-based methods. VoltageFluor (VF) indicators increase their fluorescence in response to membrane depolarizations due to attenuation of fluorescence quenching by photoinduced electron transfer (PeT). ${ }^{3}$ Within the VoltageFluor scaffold, the identity of the fluorescent reporter, the aniline electron donor, and the molecular wire can be modified to tune the spectral and voltage sensing properties of the probe..$^{4-8}$

Previously, we showed that 9',9'-dimethylfluorene (fVF, Scheme 1) can replace the phenylene-vinylene-based molecular wire in the VoltageFluor scaffold (VF2.1.Cl,

Scheme 1). ${ }^{7}$ These fluorene VoltageFluors (fVFs) are less
Scheme 1. Hybrid Vinyl-fluorene Wires for Voltage Sensing

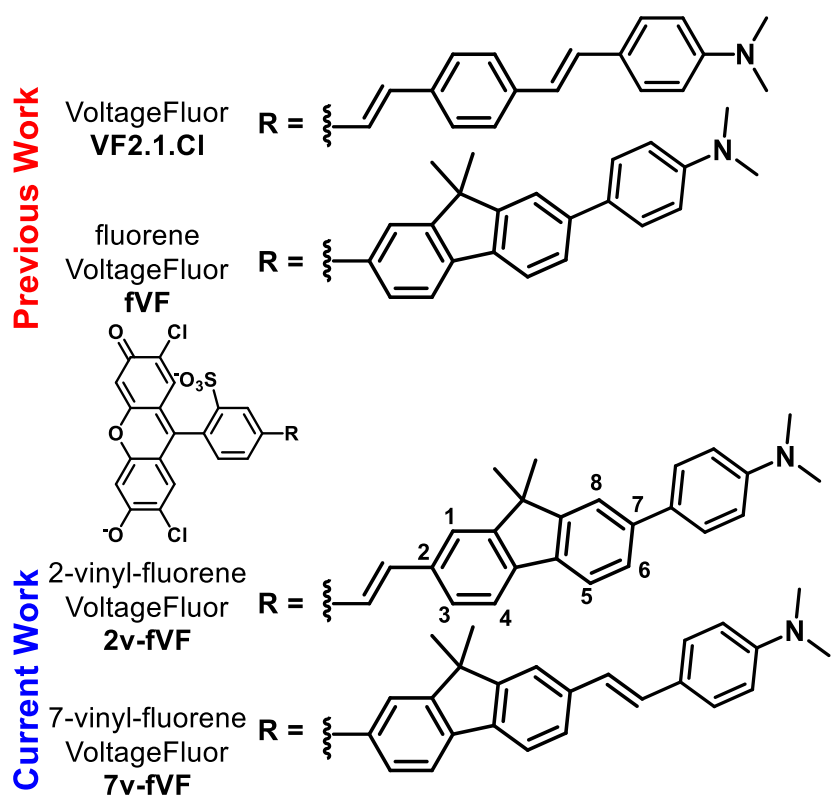

phototoxic than other voltage indicators in cardiomyocyte monolayers, making them useful for reporting cardiac 
Scheme 2. Synthesis of 2-vinyl fluorene VF dyes

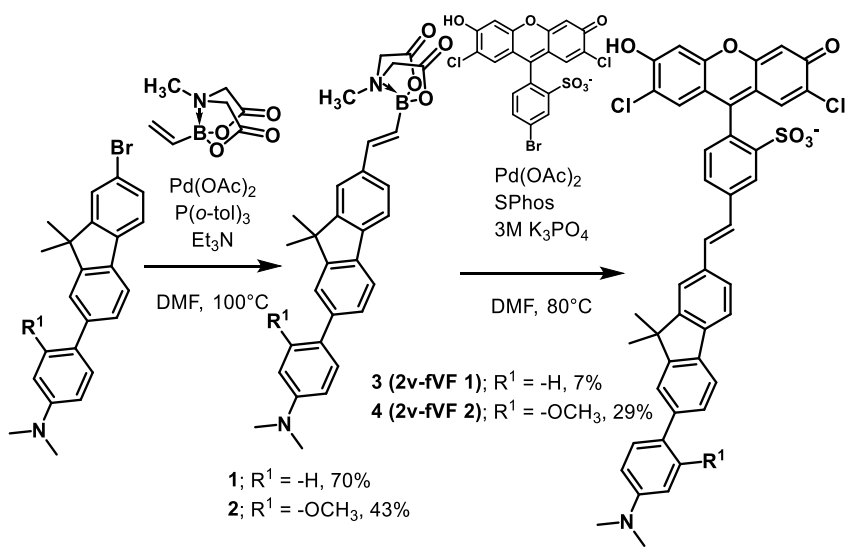

action potential kinetics over prolonged experiments. However, fVFs are 2- to 5-fold less sensitive to changes in $\mathrm{V}_{\mathrm{mem}}$ than their phenylene vinylene-based counterparts. ${ }^{7}$ We sought to improve the sensitivity of the fluorene molecular wire scaffold while harnessing its reduced phototoxicity characteristics.

We hypothesize that the voltage sensitivity of fluorene molecular wire fVF dyes is reduced due to the higher attenuation factor $\left(0.09 \AA^{-1}\right),{ }^{9}$ or $\beta$ value, of fluorenes compared to the phenylene-vinylene scaffold of $\mathrm{VF}_{2.1 .} \mathrm{Cl}(\beta=$ $\left.0.04 \AA^{-1}\right) .^{10-12}$ Previous studies showed that oligo-fluorenevinylene molecular wires, which combine aspects of fluorenes and vinyl spacers, possess a $\beta$ value of $0.075 \AA .^{13}$ Therefore, we hypothesized that incorporation of a vinylene spacer into the fluorene VoltageFluor scaffold would boost voltage sensitivity while retaining the low phototoxicity of fVF dyes (Scheme 1). These new indicators, vinylene-fluorene VoltageFluors ( $\mathrm{v}-\mathrm{fVFs}$ ), represent a structural hybrid between the two wire scaffolds reported by our laboratory.7, 14

We designed indicators with vinyl spacers in two different, regioisomeric locations: between the fluorene subunit and the dichlorofluoresein fluorophore $(2 \mathrm{~V}-\mathrm{fVFs}$, Scheme 1), and between the aniline donor and the fluorene ( $7 \mathrm{~V}^{-}$ $\mathrm{fVFs}$, Scheme 1). The numbers 2 and 7 indicate the substituent position of the vinyl group on the fluorene ring. Indicators with the $2 \mathrm{~V}-\mathrm{fVF}$ substitution are accessible in two Scheme 3. Synthesis of 7-vinyl fluorene VF dyes steps from previously reported compounds (Scheme 2, S1). ${ }^{7}$ Cross-coupling using $\mathrm{Pd}(\mathrm{OAc})_{2}$ and tri-o-tolylphosphine gives vinyl-fluorene MIDA boronate esters 1 and $\mathbf{2}$ in good yield. Suzuki-Miyaura coupling with modified conditions ${ }^{15}$ provides $2 \mathrm{~V}-\mathrm{fVF}$ indicators $3(2 \mathrm{~V}-\mathrm{fVF} 1)$ and 4 (2V-fVF 2).

Construction of regioisomeric $7 \mathrm{v}-\mathrm{fVFs}$ begins by converting substituted 4-nitrobenzaldehydes to styrenes 7 and 8 by Wittig olefination (Scheme 3). Pd-catalyzed crosscoupling with 2-bromo-7-iodo-9,9-dimethyl-9H-fluorene yields nitrostyryl-fluorene wires $\mathbf{9}$ and $\mathbf{1 0}$ as orange solids. Starting from the 4-nitrostyrenes (Scheme 3) was essential: use of the corresponding 4-aminostyrenes in the crosscoupling reaction yields the desired linear isomer in a 2:1 ratio with the branched isomer. The isomers could not be separated by chromatography. Nitrostyryl-fluorene wires are converted to anilines $\mathbf{1 1}$ and $\mathbf{1 2}$ by reduction with $\mathrm{SnCl}_{2}$, followed by reductive amination with formaldehyde to yield 13 and 14 as bright yellow solids (Scheme 3). Pd-catalyzed cross-coupling with bis-(pinacolato)diboron provides boronic esters 15 and 16 for coupling with bromo-2,7dichlorosulfofluorescein. The subsequent Pd-catalyzed cross-coupling of $\mathbf{1 5}$ or $\mathbf{1 6}$ with halogenated sulfonofluorescein furnishes $7 \mathrm{~V}$-fVF indicators $\mathbf{1 7}(7 \mathrm{~V}-\mathrm{fVF} 1)$ and $\mathbf{1 8}(7 \mathrm{~V}-\mathrm{fVF}$ 2). As control compounds, we also constructed indicators that lack an aniline donor (Scheme S2, 2v-fVF o and $\mathbf{7 v -}$ fVF o).

Spectroscopic characterization of the six new indicators reveal that the position of the vinyl spacer has no effect on the absorption or emission of the xanthene fluorophore (Figure S1, Table S1). However, relative to fVF dyes, both of the vinyl-fluorene substitution patterns result in bathochromic shifts in the molecular wire absorbance. This shift is larger for $7 \mathrm{v}$-fVF substitution (Figure S1, Table $\mathbf{S} \mathbf{1}$ ). The red shift of the molecular wire absorbance is due to the increased conjugation in the molecular wire system, which is electronically decoupled from the xanthene chromophore in the ground state. In agreement with a PeT mechanism for voltage sensing, indicators lacking an aniline exhibit quantum yields two to three times higher than the donorcontaining counterparts (Table S1).

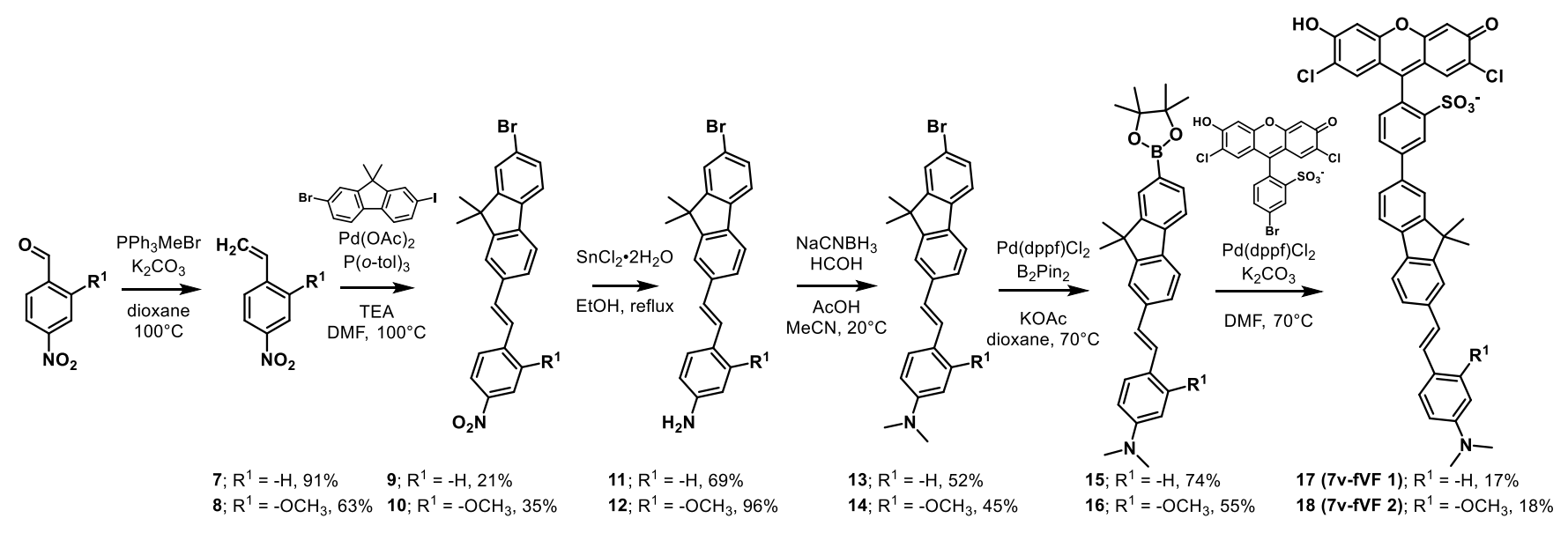



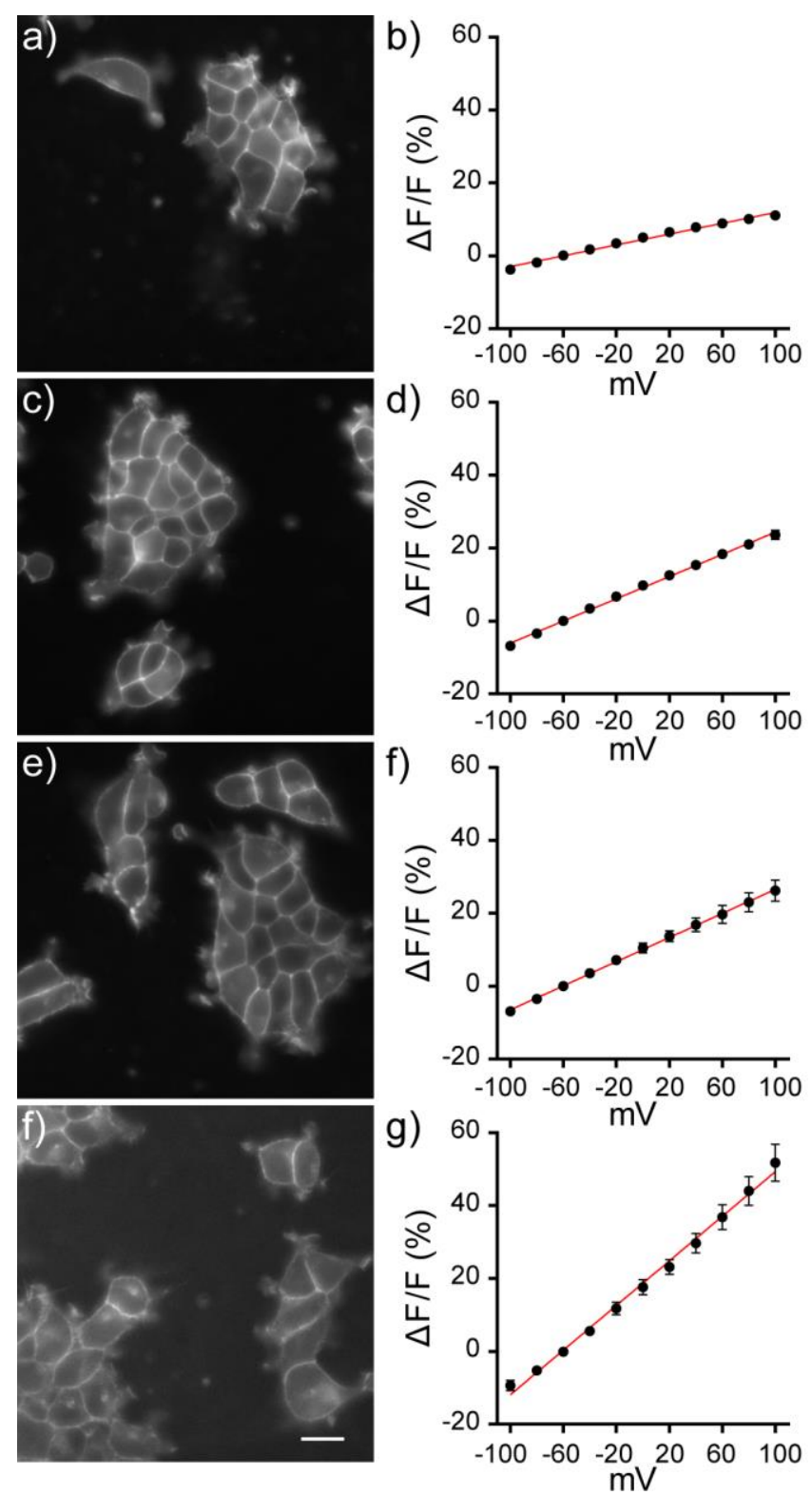

Figure 1. Characterization of vinyl-fluorene VoltageFluors in HEK293T cells. Live cell fluorescence images of a) $2 \mathrm{~V}-\mathrm{fVF} 1, \mathbf{c})$ $2 \mathrm{v}-\mathrm{fVF} 2$, e) $7 \mathrm{~V}-\mathrm{fVF} 1$, and f) $7 \mathrm{v}$-fVF 2 loaded at $0.5 \mu \mathrm{M}$ in HEK293T cells. Scale bar is $20 \mu \mathrm{m}$. Plots of $\Delta \mathrm{F} / \mathrm{F}$ versus membrane potential (millivolts) in voltage-clamped HEK293T cells for b) $2 \mathrm{v}-\mathrm{fVF} 1, \mathbf{d}$ ) $2 \mathrm{v}-\mathrm{fVF} 2, \mathbf{f}) 7 \mathrm{v}-\mathrm{fVF} 1$, and g) $7 \mathrm{v}$-fVF 2 . The red line is the line of best fit, error bars are \pm SEM for a minimum of 3 independent determinations.

To test the effect of vinyl spacers on voltage sensitivity, we recorded the fluorescence intensity of voltage-clamped HEK293T cells stained with vinyl-fluorene VoltageFluors. Indicators with the 2-vinyl spacer (2v-fVF 1 and 2V-fVF 2) exhibit a 7.4 and $15.2 \% \Delta \mathrm{F} / \mathrm{F}$ per $100 \mathrm{mV}$, respectively (Figure 1, Table 1). When compared to the respective fluorene VoltageFluors (fVF dyes), ${ }^{7}$ this structural modification produces a modest $60 \%$ and $40 \%$ improvement to sensitivity (fVF 1, 4.5\%; fVF 2, 10.5\% $\Delta \mathrm{F} / \mathrm{F}$ per $100 \mathrm{mV}$ change, Figure S2). ${ }^{7}$ The 7 -vinyl spacer improves voltage sensitivity to a greater extent; $\mathbf{7 v - f V F} 1$ has a $\mathbf{1 6 . 5} \% \Delta \mathrm{F} / \mathrm{F}$ (350\% improvement) and $7 \mathbf{v}-\mathbf{f V F} 2$ exhibits a $30.6 \% \Delta \mathrm{F} / \mathrm{F}(290 \%$
Table 1. Voltage Sensitivity of v-fVF dyes

\begin{tabular}{|c|c|c|c|}
\hline Entry & $\begin{array}{c}\text { Bright- } \\
\text { ness }^{\mathrm{a}}\end{array}$ & $\% \Delta \mathrm{F} / \mathrm{F}^{\mathrm{b}}$ & SNR \\
\hline \hline 2V-fVF 1 (3) & 1.6 & $7.4 \pm 0.3$ & $41 \pm 10.5$ \\
\hline 7V-fVF 1 (17) & 1.0 & $16.5 \pm 0.2$ & $67 \pm 8.5$ \\
\hline 2V-fVF 2 (4) & 1.5 & $15.2 \pm 0.2$ & $49 \pm 3.4$ \\
\hline 7V-fVF 2 (18) & 0.4 & $30.6 \pm 0.7$ & $37 \pm 5.6$ \\
\hline 2V-fVF o (20) & 5.8 & $-0.3 \pm 0.01$ & $1.7 \pm 0.2$ \\
\hline 7V-fVF o (23) & 4.4 & $-0.3 \pm 0.01$ & $0.8 \pm 0.2$ \\
\hline
\end{tabular}

${ }^{\mathrm{a}}$ Measured in HEK 293T cells. ${ }^{\mathrm{b}}$ per $100 \mathrm{mV}$, recorded at 0.5 $\mathrm{kHz}$ optical sampling rate.

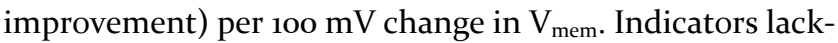
ing an aniline donor (2V-fVF o and $\mathbf{7 V - f V F ~ o ) ~ w e r e ~ n o t ~ s e n - ~}$ sitive to changes in $\mathrm{V}_{\text {mem }}$, consistent with a PeT-based mechanism of voltage sensing (Figure S2g-i, p-r, Table 1)

In rat hippocampal neurons, all of the new v-fVF indicators have higher $\Delta \mathrm{F} / \mathrm{F}$ and signal-to-noise (SNR) per action potential than $\mathrm{fVF} 2$, the best of the fluorene-only fVF series. ${ }^{7}$ Of the new indicators, $7 \mathbf{v}$-fVF 1 (17) has the highest SNR per action potential, at 18:1, while $7 \mathbf{v}-\mathbf{f V F} 2(\mathbf{1 8})$ has the highest $\Delta \mathrm{F} / \mathrm{F}$, at $9 \%$ per action potential (Table $\mathbf{S 2}$, Figure $\left.S_{3}\right)$. However, under identical conditions, phenylenevinylene-based $\mathrm{VF}^{2.1}$. Cl retains the best SNR per action potential, at 35:1.

In spontaneously contracting human induced pluripotent stem cell-derived cardiomyocyte (hiPSC-CM) monolayers, all of the new $\mathrm{v}$-fVF indicators report cardiac action potential kinetics with high SNR. Vinyl-fVF indicators have larger SNR per action potential than either fVF 2 or VF2.1.Cl, albeit with a lower $\Delta \mathrm{F} / \mathrm{F}$ (Table S2, Figure 2, Figure $\left.S_{4}\right)$. Previously, we found that substituting the phenylene-vinylene molecular wire $\left(\mathrm{VF}^{2.1 .} \mathrm{Cl}\right)$ with a fluorene monomer (fVF) in the VF scaffold substantially reduces phototoxicity in cardiomyocytes, allowing up to ten minutes of continuous illumination without disrupting the waveform of action potentials. ${ }^{7}$ Thus, we were curious whether the new vinyl-fluorene hybrids, with their enhanced voltage sensitivity compared to fluorene-only indicators, would behave more like fVF 2 or $\mathrm{VF} 2.1 \mathrm{Cl}$ in terms of phototoxicity. We continuously illuminated hiPSC-CM monolayers stained with indicators, making ten second recordings every minute to monitor action potential morphology. Indicators with $2 \mathrm{v}$-fVF substitution appear extremely phototoxic-action potential morphology changes after just one (2v-fVF 1, Figure $3 \mathbf{a})$ to three (2v-fVF 2, Figure $3 \mathbf{b})$ minutes of illumination. Shortly after, the hiPSCCMs stopped beating (Figure $\mathbf{3 a}, \mathbf{b}$ ). This degree of toxicity was similar to $\mathrm{VF}_{2.1} \mathrm{Cl}$ (Figure $\boldsymbol{S}_{5}$ ).

However, placing the vinyl spacer at the 7-position results in indicators that are much less phototoxic than either $2 \mathrm{~V}$-fVF or VF2.1.Cl (Figure 3c-f). With either $\mathbf{7 v}$-fVF 1 (Figure 3c) or $\mathbf{7 v - f V F} 2$ (Figure 3d), recordings can be 

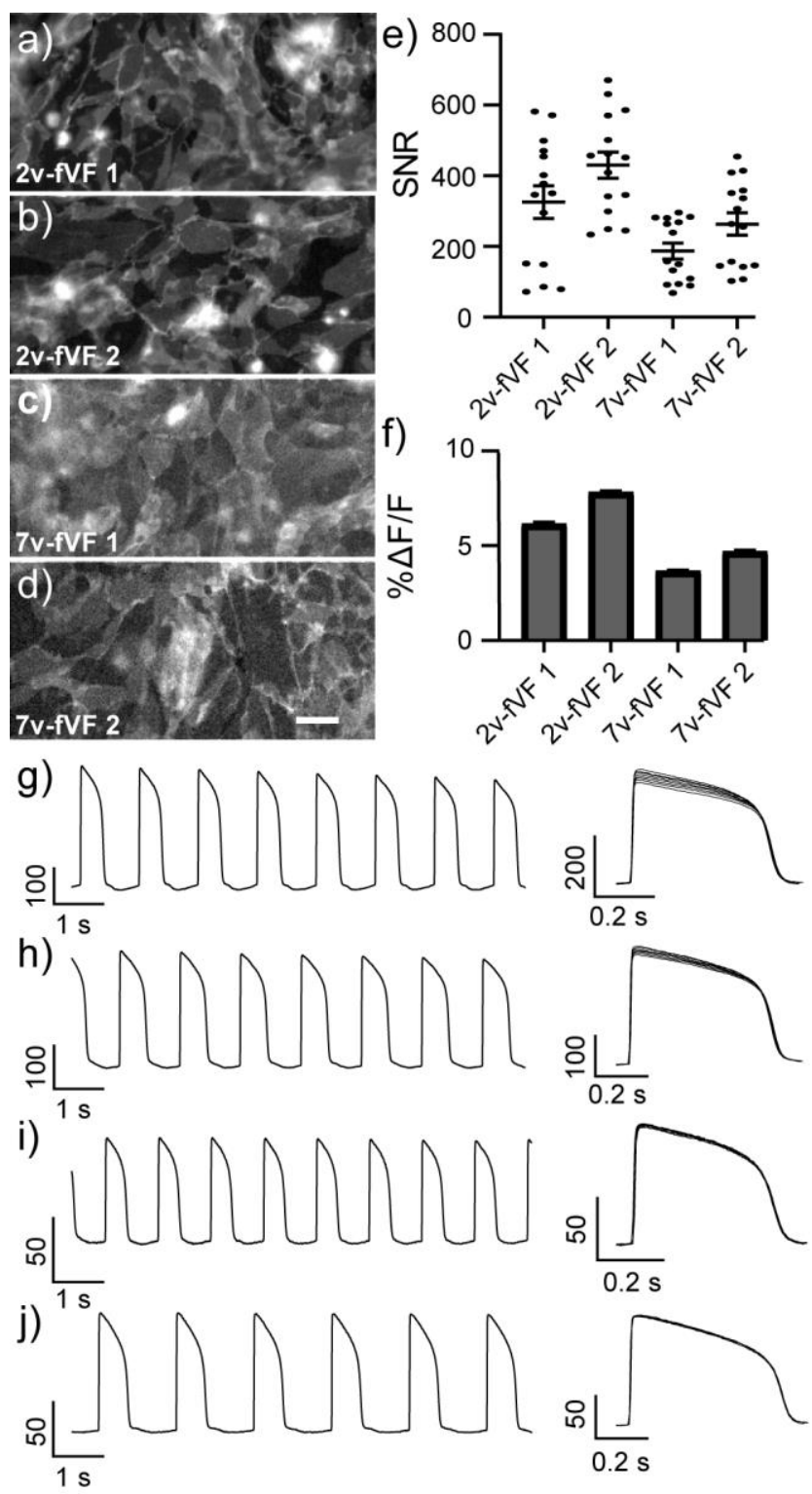

Figure 2. Performance of vinyl-fluorene VoltageFluors in hiPSC-CM monolayers. Representative fluorescence micrograph of hiPSC-CMs loaded with $0.5 \mu \mathrm{M}$ of either a) $2 \mathrm{~V}$-fVF 1 (3), b) $2 \mathrm{~V}-\mathrm{fVF} 2$ (4), c) $7 \mathrm{v}-\mathrm{fVF} 1$ (17), or d) $7 \mathrm{v}$-fVF 2 (18). Scale bar is $50 \mu \mathrm{m}$. e) Plot of SNR per action potential for each indicator. Data are mean \pm S.E.M. f) Plot of $\Delta F / F$ per action potential in hiPSC-CMs for each indicator. Data are mean \pm S.E.M. from $n=15$ recordings. $g-j$ ) left Plots of fluorescence intensity vs. time for either g) $2 \mathrm{~V}$-fVF 1 (3), h) $2 \mathrm{~V}$-fVF 2 (4), i) $7 \mathrm{~V}$-fVF 1 (17), or j) $7 \mathrm{~V}-\mathrm{fVF} 2$ (18). right Concatenated action potentials.

made for up to 10 minutes without any change to action potential shape (Figure 3, Figure $\mathbf{S}_{\mathbf{5}}$ ). Unlike fluoreneonly fVF 2 , the SNR remains high for both $7 \mathbf{v}$-fVF 1 and $7 \mathbf{v}$ fVF 2 throughout the duration of the experiments-above 100:1 after illuminating $\mathbf{7 v - f V F} \mathbf{2}$ for 9 minutes-permitting long-term recordings of electrical activity in cardiomyocyte monolayers (Figure $\boldsymbol{S}_{\mathbf{5}}$ ).

The precise molecular mechanisms governing the substantially reduced phototoxicity of the $7 \mathrm{v}$-fVF scaffold compared to $2 \mathrm{~V}$-fVF and $\mathrm{VF}$ 2.1. $\mathrm{Cl}$ remain elusive. One a) $0 \min \quad 2 \min \quad 5 \min \quad 10 \min$
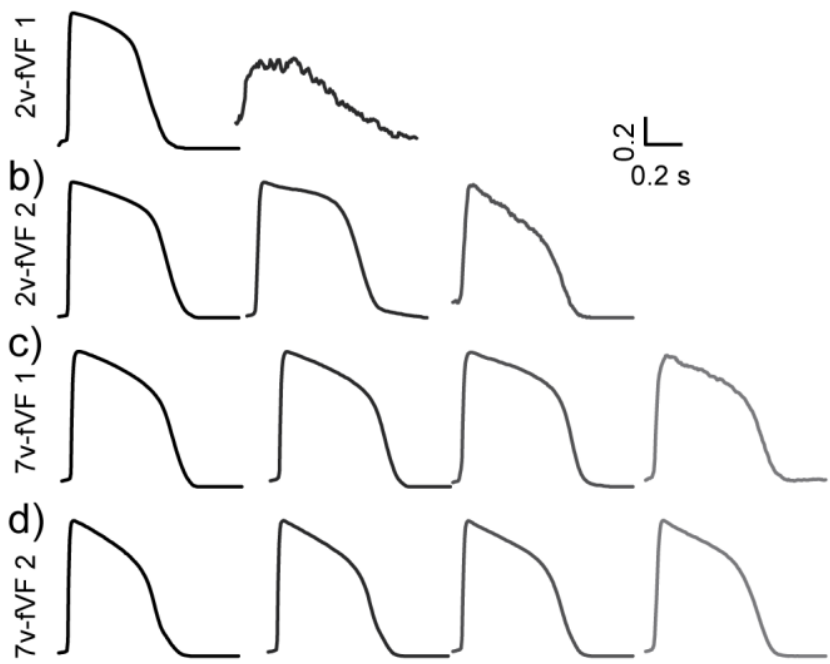

e)

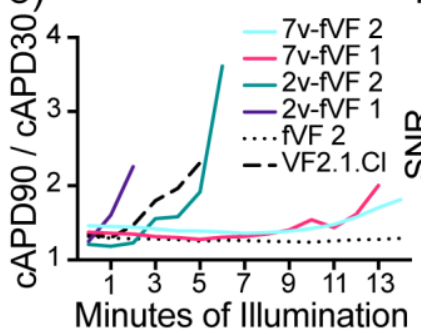

f)

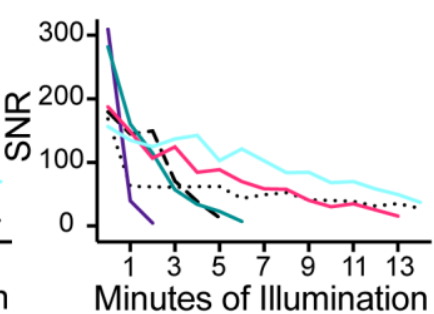

Figure 3. Extended imaging with vinyl-fluorene VoltageFluors in hiPSC-iCM monolayers under constant illumination. Monolayers were stained with v-fVF indicators and illuminated constantly. Every minute, 1o second optical recordings of voltage dynamics were acquired. Representative traces are shown for a) $2 \mathrm{v}$-fVF 1 (3), b) $2 \mathrm{~V}-\mathrm{fVF} 2$ (4), c) $7 \mathrm{v}-\mathrm{fVF} 1(17)$, and d) $7 \mathrm{v}$ fVF 2 (18). e) Plot of the ratio of cAPD9o/cAPD30, which is the action potential duration at 90 and 30 percent of the repolarization, corrected for beat rate by Fridericia's formula. Initial recordings start with a ratio between 1.2 and 1.4, regardless of the indicator used. Increases in the ratio indicate a change in action potential morphology. f) Plot of SNR per action potential vs. total minutes of illumination.

hypothesis is that the dyes accumulate differently in cellular membranes, leading to higher effective concentrations for one dye compared to another. This might result in an increased rate of reactive oxygen species (ROS) production, leading to cell death. Indeed, phototoxicity of many voltage indicators can be decreased by lowering the concentration used to stain cells. ${ }^{7}{ }^{16-18}$ Although v-fVF dyes show some small differences in cellular brightness (Figure S6, Table 1), fluorescence intensity alone cannot be used as a quantitative measure of dye concentration. Rates of photobleaching do not correlate with phototoxicity. Although the less toxic $7 \mathrm{v}$-fVF indicators have slower rates of photobleaching compared to 2V-fVF dyes (in HEK293T cells, Figure $S_{7}$ ), this correlation does not always hold: the less phototoxic fVF 2 bleaches more rapidly than $\mathrm{VF2.1.Cl}$ (Figure $\mathrm{S}_{7}$ ). Another hypothesis is that the more phototoxic $2 \mathrm{v}$-fVF dyes position the vinyl group at the lipid/aqueous interface on the surface of the cell, ${ }^{14}$ leading to greater exposure to oxygen and increasing the likelihood 
of either sensitizing molecular oxygen or reacting with singlet oxygen, a primary decomposition pathway for phenylenevinylene structures. ${ }^{19}$ In contrast, the less phototoxic $7 \mathrm{~V}$-fVF dyes bury the vinyl spacer in the lipid bilayer of the cellular membrane, shielding it from oxygen that might contribute both to phototoxicity and photobleaching. Studies are under way to probe these mechanisms and hypotheses in greater detail.

In summary, we developed four new voltage indicators. All four new vinylene-fluorene VoltageFluor (v-fVFs) indicators show higher voltage sensitivity $(\Delta \mathrm{F} / \mathrm{F})$ than their cognate fluorene VoltageFluor dyes (fVFs). We detail the synthesis and characterization of these indicators in HEK293T cells, mammalian neurons, and human iPSC-derived cardiomyocytes to demonstrate the effect of this small structural change to the molecular wire on the performance of the voltage indicator. We found that adding a vinyl spacer between the electron donating aniline and the fluorene in the molecular wire ( $7 \mathrm{~V}-\mathrm{fVFs})$ increased sensitivity by about 3-fold compared to fVF dyes. For $7 \mathbf{v}$-fVF 2 (18), voltage sensitivity was higher $(30 \% \Delta \mathrm{F} / \mathrm{F})$ than the sensitivity of $\mathrm{VF} 2.1 . \mathrm{Cl}(25 \% \Delta \mathrm{F} / \mathrm{F})$. More importantly, even with improved sensitivity, $7 \mathrm{v}$-fVF indicators remain less phototoxic in cardiomyocytes relative to $\mathrm{VF2.1.Cl}$, while maintaining higher SNR throughout extended recordings. Vinyl-fluorene VoltageFluors represent an improvement over the fluorene VoltageFluor scaffold and are an attractive alternative to the traditional VoltageFluor scaffold for prolonged recordings of electrical activity in cells.

\section{ASSOCIATED CONTENT}

\section{Supporting Information.}

Supplementary data, including supporting figures, spectra, procedures, and analysis. This material is available free of charge via the Internet at http://pubs.acs.org.

\section{AUTHOR INFORMATION}

\section{Corresponding Author}

* Evan W. Miller, evanwmiller@berkeley.edu

\section{ACKNOWLEDGMENT}

We thank the NIH for support of this research (R35GM119855). S.C.B. was supported in part by a grant from the NIH (T32GMo66698).

\section{REFERENCES}

1. Peterka, D. S.; Takahashi, H.; Yuste, R., Imaging Voltage in Neurons. Neuron 2011, 69 (1), 9-21.

2. Liu, P.; Miller, E. W., Electrophysiology, Unplugged: Imaging Membrane Potential with Fluorescent Indicators. Accounts of Chemical Research 2020, 53 (1), 11-19.

3. Miller, E. W.; Lin, J. Y.; Frady, E. P.; Steinbach, P. A.; Kristan, W. B., Jr.; Tsien, R. Y., Optically monitoring voltage in neurons by photo-induced electron transfer through molecular wires. Proc Natl Acad Sci U S A 2012, 109 (6), 2114-9.

4. Woodford, C. R.; Frady, E. P.; Smith, R. S.; Morey, B.; Canzi, G.; Palida, S. F.; Araneda, R. C.; Kristan, W. B., Jr.; Kubiak, C. P.; Miller, E. W.; Tsien, R. Y., Improved PeT molecules for optically sensing voltage in neurons. J Am Chem Soc 2015, 137 (5), 1817-24.
5. Deal, P. E.; Kulkarni, R. U.; Al-Abdullatif, S. H.; Miller, E. W., Isomerically Pure Tetramethylrhodamine Voltage Reporters. JAm Chem Soc 2016, 138 (29), 9085-8.

6. Ortiz, G.; Liu, P.; Naing, S. H. H.; Muller, V. R.; Miller, E. W., Synthesis of Sulfonated Carbofluoresceins for Voltage Imaging. Journal of the American Chemical Society 2019, 141 (16), 6631-6638.

7. Boggess, S. C.; Gandhi, S. S.; Siemons, B. A.; Huebsch, N.; Healy, K. E.; Miller, E. W., New Molecular Scaffolds for Fluorescent Voltage Indicators. ACS chemical biology 2019, 14 (3), 390-396.

8. Boggess, S. C.; Lazzari-Dean, J. R.; Raliski, B. K.; Mun, D. M.; Li, A. Y.; Turnbull, J. L.; Miller, E. W., Fluorescence lifetime predicts performance of voltage sensitive fluorophores in cardiomyocytes and neurons. RSC Chemical Biology 2021, 2 (1), 248-258.

9. $\quad$ Goldsmith, R. H.; Sinks, L. E.; Kelley, R. F.; Betzen, L. J.; Liu, W.; Weiss, E. A.; Ratner, M. A.; Wasielewski, M. R., Wire-like charge transport at near constant bridge energy through fluorene oligomers. Proceedings of the National Academy of Sciences of the United States of America 2005, 102 (10), 3540-3545.

10. Davis, W. B.; Svec, W. A.; Ratner, M. A.; Wasielewski, M. R., Molecular-wire behaviour in p -phenylenevinylene oligomers. Nature 1998, 396 (6706), 6o-63.

11. Eng, M. P.; Albinsson, B., Non-exponential distance dependence of bridge-mediated electronic coupling. Angew Chem Int Ed Engl 2006, 45 (34), 5626-5629.

12. Albinsson, B.; Mårtensson, J., Long-range electron and excitation energy transfer in donor-bridge-acceptor systems. Journal of Photochemistry and Photobiology C: Photochemistry Reviews 2008, 9 (3), 138-155.

13. Wielopolski, M.; Santos, J.; Illescas, B. M.; Ortiz, A.; Insuasty, B.; Bauer, T.; Clark, T.; Guldi, D. M.; Martín, N., Vinyl spacers-tuning electron transfer through fluorene-based molecular wires. Energy E Environmental Science 2o11, 4 (3), 765771.

14. Kulkarni, R. U.; Yin, H.; Pourmandi, N.; James, F.; Adil, M. M.; Schaffer, D. V.; Wang, Y.; Miller, E. W., A Rationally Designed, General Strategy for Membrane Orientation of Photoinduced Electron Transfer-Based Voltage-Sensitive Dyes. ACS chemical biology 2017, 12 (2), 407-413.

15. Uno, B. E.; Gillis, E. P.; Burke, M. D., Vinyl MIDA boronate: a readily accessible and highly versatile building block for small molecule synthesis. Tetrahedron 2oo9, 65 (16), 3130-3138.

16. McKeithan, W. L.; Savchenko, A.; Yu, M. S.; Cerignoli, F.; Bruyneel, A. A. N.; Price, J. H.; Colas, A. R.; Miller, E. W.; Cashman, J. R.; Mercola, M., An Automated Platform for Assessment of Congenital and Drug-Induced Arrhythmia with hiPSC-Derived Cardiomyocytes. Frontiers in physiology 2017, 8, 766.

17. McPheeters, M. T.; Wang, Y. T.; Werdich, A. A.; Jenkins, M. W.; Laurita, K. R., An infrared optical pacing system for screening cardiac electrophysiology in human cardiomyocytes. PLOS ONE 2017, 12 (8), eo183761.

18. Rohr, S.; Salzberg, B. M., Multiple site optical recording of transmembrane voltage (MSORTV) in patterned growth heart cell cultures: assessing electrical behavior, with microsecond resolution, on a cellular and subcellular scale. Biophysical Journal 1994, 67 (3), 1301-1315.

19. Zheng, Q.; Jockusch, S.; Zhou, Z.; Blanchard, S. C., The contribution of reactive oxygen species to the photobleaching of organic fluorophores. Photochemistry and photobiology 2014, 90 (2), 448-454. 


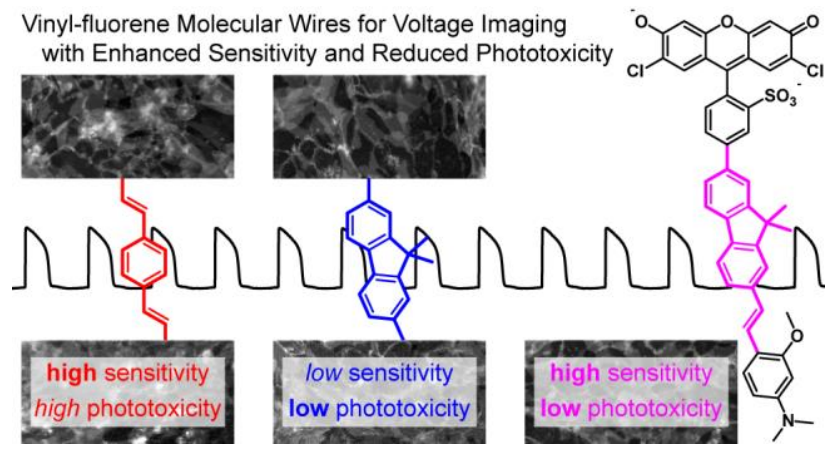

